\title{
Evolutionary complexification of plant information structure under influence of permanent data flow
}

\author{
Moldavanov A. \\ Belkin International Inc., Los Angeles, USA \\ email: trandrei8@gmail.com
}

\begin{abstract}
Numerical and analytical simulation targeting a process of complexification for information structure of plant's subsystem is conducted. Plant's subsystem is considered as an open thermodynamic system and simulation employs the mathematical model utilizing formalism of randomized continuity equation. Approximation of open system is achieved by use of infinite number of links with environment in assumption that all links are executed by continuity equation for information exchange. Following this approach we deal with a phase space at determined exchange rate and random interface factor. Found solution is a function of an information interface factor and in its physical meaning is an integral efficiency of information exchange. Executed formalism assumes natural extension to computation methods based on binomial probability distribution. Discovered solution possesses the built-in mechanism for phasing (existence of the stages of dissimilar physical meaning), dynamic transformation of topology for information exchange, predetermined change of range for random variations, and existence of specific fluctuations for information components of complexification process. Verification of results is conducted by numerical methods.
\end{abstract}

